\title{
Cooper Pair with Nonzero Momentum in System with Spin Dependent Mass of Quasiparticles
}

\author{
J. KaCZMARCZYK AND J. SPAŁEK* \\ Marian Smoluchowski Institute of Physics, \\ Jagiellonian University, Reymonta 4, 30-059 Kraków, Poland
}

We consider a single Cooper pair with the spin dependent quasiparticle masses, with and without applied magnetic field. Such situation takes place for the strongly correlated electron systems, where a relatively strong Hubbard interaction differentiates the quasiparticle states in the majorityand the minority-spin subbands. In that situation, the two spin subbands in an applied magnetic field are not only shifted one with respect to the other, but also distorted differently, which results from the electronic correlations. Under these circumstances, the fermionic particles composing the Cooper pair, are quantum mechanically distinguishable. In result, the Cooper pair has a nonzero momentum (i.e. produces a stationary current in a similar manner as electron in the Bloch state), and there exists a critical value of the attractive interaction, below which the bound Cooper-pair state is not formed. The presence of the applied field (included via the Zeeman term) does not alter the picture qualitatively. Importance of these results for the formation of the Fulde-Ferrell-Larkin-Ovchinnikov phase in strongly correlated systems is mentioned.

PACS numbers: 71.24.+q, 71.10.Li

\section{Introduction}

The Cooper pair bound state [1] represents one of standard two-particle problems in quantum mechanics when we have an electron gas present as a background.

In the situation with strong Coulomb interaction, the quasiparticle mass in magnetic field depends on the spin orientation. Namely, electrons in the spin-majority $(\sigma=\uparrow)$ band have the quasiparticle mass $m_{1} \equiv m_{\uparrow}$ different than the electrons with their spin in the opposite direction, $m_{2} \equiv m_{\downarrow}[2,3]$. This situation takes place for instance in the CeCoIn 5 superconductor [4]. A theory of superconductivity which would take account of spin dependent masses could be helpful in describing such systems.

*corresponding author; e-mail: ufspalek@if.uj.edu.pl 
In this paper we describe the Cooper pair with the spin dependent masses of quasiparticles by means of modified Cooper approach, which provides a starting point for the modified BCS theory of condensed superconducting state. We first summarize the standard Cooper approach to the two-particle pairing problem. Then, in Sect. 2, we describe how the dependence of masses on spin direction changes the Landau-Fermi liquid theory and solve the Cooper problem with the spin dependent masses. Numerical results obtained from this solution are provided in Sect. 3. Finally, we summarize briefly our work and provide an outlook in Sect. 4 .

\subsection{Cooper pair - a brief summary}

We consider a Fermi sea of noninteracting electrons, to which we add two interacting electrons. The Schrödinger equation for such a system is as follows:

$$
H \Psi=E \Psi
$$

with the Hamiltonian

$$
H=-\frac{\hbar^{2}}{2 m} \nabla_{1}^{2}-\frac{\hbar^{2}}{2 m} \nabla_{2}^{2}+V\left(\boldsymbol{r}_{1}-\boldsymbol{r}_{2}\right) .
$$

The pair wave function is represented as a superposition of the wave functions of noninteracting electrons in volume $V$ :

$$
\Psi\left(\boldsymbol{r}_{1}, \boldsymbol{r}_{2} ; \sigma_{1}, \sigma_{2}\right)=\frac{1}{V} \sum_{\boldsymbol{k}_{1}, \boldsymbol{k}_{2}} \alpha_{\boldsymbol{k}_{1}, \boldsymbol{k}_{2}} \mathrm{e}^{\mathrm{i} \boldsymbol{k}_{1} \boldsymbol{r}_{1}+\mathrm{i} \boldsymbol{k}_{2} \boldsymbol{r}_{2}} \chi\left(\sigma_{1}, \sigma_{2}\right),
$$

where $\chi\left(\sigma_{1}, \sigma_{2}\right)$ describes the spin-singlet state. To simplify the calculations we make the transformation to the center of mass and relative coordinates, i.e. select the center-of-mass variables

$$
\boldsymbol{R}=\frac{\boldsymbol{r}_{1}+\boldsymbol{r}_{2}}{2}, \quad \boldsymbol{Q}=\boldsymbol{k}_{1}+\boldsymbol{k}_{2},
$$

as well as those representing the relative motion of particles

$$
\boldsymbol{r}=\boldsymbol{r}_{1}-\boldsymbol{r}_{2}, \quad \boldsymbol{k}=\frac{\boldsymbol{k}_{1}-\boldsymbol{k}_{2}}{2} .
$$

Obviously, they correspond to the real- and reciprocal-space variables, respectively. The real-space pair wave function is then selected in the form

$$
\Psi(\boldsymbol{R}, \boldsymbol{r})=\frac{1}{V} \mathrm{e}^{\mathrm{i} \boldsymbol{Q R}} \sum_{\boldsymbol{k}} \alpha_{\boldsymbol{k}} \mathrm{e}^{\mathrm{i} \boldsymbol{r} \boldsymbol{r}},
$$

where we have extracted from the summation the center-of-mass part, as the total momentum $\boldsymbol{Q}$ is conserved. We now use the Schrödinger equation and assume that the pairing potential in $\boldsymbol{k}$ space: $V_{\boldsymbol{k}, \boldsymbol{k}^{\prime}} \equiv \frac{N}{V} \int_{V} \mathrm{e}^{-\mathrm{i} \boldsymbol{k} \boldsymbol{r}} V(\boldsymbol{r}) \mathrm{e}^{\mathrm{i} \boldsymbol{k}^{\prime} \boldsymbol{r}} \mathrm{d}^{3} r$, is attractive $\left(\equiv-V_{0}\right)$ at and above the Fermi surface (in the energy regime of width $\left.\hbar \omega_{\mathrm{D}}\right)$ and zero elsewhere.

\subsection{Pair binding energy}

Following the standard Cooper procedure, we obtain the self-consistent equation for the binding energy $\Delta$ in the form 


$$
\frac{N}{V_{0}}=\sum_{\boldsymbol{k} \in \bar{W}_{\boldsymbol{Q}}} \frac{1}{\epsilon_{\boldsymbol{Q}}+\epsilon_{\boldsymbol{k}}-2 \epsilon_{\mathrm{F}}+\Delta}
$$

where $\epsilon_{\boldsymbol{Q}} \equiv \frac{\hbar^{2}}{2 M} \boldsymbol{Q}^{2}$ is the center-of-mass kinetic energy, $\epsilon_{\boldsymbol{k}} \equiv \frac{\hbar^{2}}{2 \mu} \boldsymbol{k}^{2}$ is that of relative motion, and $\epsilon_{\mathrm{F}}$ is the Fermi energy. Additionally, $\bar{W}_{\boldsymbol{Q}}$ is the range of integration dependent on the pair center-of-mass momentum $Q$ (cf. Fig. 2). In the case of equal quasiparticle masses the stationary pair with $\boldsymbol{Q}=0$ has the maximum binding energy. The range of integration is spherically symmetric, therefore we obtain an analytic solution in the well-known form [1]

$$
\Delta=\frac{2 \hbar \omega_{\mathrm{D}}}{\exp \left(\frac{2}{V_{0} \rho\left(\epsilon_{\mathrm{F}}\right)}\right)-1} \approx 2 \hbar \omega_{\mathrm{D}} \exp \left(-\frac{2}{V_{0} \rho\left(\epsilon_{\mathrm{F}}\right)}\right) .
$$

An important thing to note is that the electrons bind only if the density of states $\rho\left(\epsilon_{\mathrm{F}}\right) \neq 0$. Therefore, the background electronic gas plays a passive, but essential role in having $\Delta>0$. In the next section this role is amplified and qualitatively changed by the fact that the masses are different, $m_{\uparrow}<m_{\downarrow}$.

\section{Cooper pair with spin dependent masses}

In such a gas we deal with two types of particles: $m_{1}>m_{2}$. Therefore, there are also two dispersion relations

$$
\epsilon_{\boldsymbol{k}_{1}}=\frac{\hbar^{2}}{2 m_{1}} \boldsymbol{k}_{1}^{2}, \quad \epsilon_{\boldsymbol{k}_{2}}=\frac{\hbar^{2}}{2 m_{2}} \boldsymbol{k}_{2}^{2} .
$$

The Fermi energies for the two types of quasiparticles have to be equal. This condition together with (9) yields the relation $k_{\mathrm{F} 1}=k_{\mathrm{F} 2} \sqrt{m_{1} / m_{2}}$, which means that unequal masses lead to the different radii of the Fermi spheres (cf. Fig. 1).

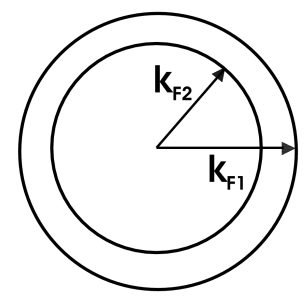

Fig. 1. Fermi spheres for the two spin subbands with different effective mass of the corresponding particles and $\boldsymbol{Q}=\mathbf{0}$.

This approach may seem unrealistic, because we did not include a Zeeman term, which is essential in the process of mass splitting. However, there could exist systems in which the quasiparticle mass is split even without an applied magnetic field (for example the quark systems [5]). If we want to include magnetic field in our calculations, the dispersion relations become shifted one with respect to the 
other by twice the Zeeman term ${ }^{\dagger}$

$$
\left\{\begin{array}{l}
\epsilon_{\boldsymbol{k}_{1}}=\frac{\hbar^{2}}{2 m_{1}} \boldsymbol{k}_{1}^{2}-g \mu_{\mathrm{B}} \frac{\hbar}{2} B \\
\epsilon_{\boldsymbol{k}_{2}}=\frac{\hbar^{2}}{2 m_{2}} \boldsymbol{k}_{2}^{2}+g \mu_{\mathrm{B}} \frac{\hbar}{2} B
\end{array}\right.
$$

\subsection{Cooper problem with spin-split masses}

We tackle this problem starting from Hamiltonian

$$
H=-\frac{\hbar^{2}}{2 m_{1}} \nabla_{1}^{2}-\frac{\hbar^{2}}{2 m_{2}} \nabla_{2}^{2}-g \mu_{\mathrm{B}} B\left(S_{1}^{z}+S_{2}^{z}\right)+V\left(\boldsymbol{r}_{1}-\boldsymbol{r}_{2}\right),
$$

which consists of the interaction $V\left(\boldsymbol{r}_{1}-\boldsymbol{r}_{2}\right)$ and the noninteracting part giving dispersion relations of the form (10) or (9), the latter in the situation with the applied field $\boldsymbol{B}=\mathbf{0}$.

The pair wave function is, just as before, a superposition of wave functions of noninteracting particles

$$
\Psi\left(\boldsymbol{r}_{1}, \boldsymbol{r}_{2} ; \sigma_{1}, \sigma_{2}\right)=\frac{1}{V} \sum_{\boldsymbol{k}_{1}, \boldsymbol{k}_{2}} \alpha_{\boldsymbol{k}_{1}, \boldsymbol{k}_{2}} \mathrm{e}^{\mathrm{i} \boldsymbol{k}_{1} \boldsymbol{r}_{1}+\mathrm{i} \boldsymbol{k}_{2} \boldsymbol{r}_{2}} \chi\left(\sigma_{1}, \sigma_{2}\right)
$$

To solve the problem we make the transformation to the center-of-mass and relative coordinates which now looks a little differently (cf. Wróbel et al. [1])

$$
\boldsymbol{R}=\frac{\boldsymbol{r}_{1} m_{1}+\boldsymbol{r}_{2} m_{2}}{m_{1}+m_{2}}, \quad \boldsymbol{Q}=\boldsymbol{k}_{1}+\boldsymbol{k}_{2}
$$

and

$$
\boldsymbol{r}=\boldsymbol{r}_{1}-\boldsymbol{r}_{2}, \quad \boldsymbol{k}=\frac{\boldsymbol{k}_{1} m_{2}-\boldsymbol{k}_{2} m_{1}}{m_{1}+m_{2}}
$$

The transformed wave function and Hamiltonian are as follows, respectively:

$$
\begin{aligned}
& \Phi(\boldsymbol{R}, \boldsymbol{r})=\frac{1}{V} \mathrm{e}^{\mathrm{i} \boldsymbol{Q} \boldsymbol{R}} \sum_{\boldsymbol{k}} \alpha_{\boldsymbol{k}} \mathrm{e}^{\mathrm{i} \boldsymbol{k} \boldsymbol{r}}, \\
& H=-\frac{\hbar^{2}}{2 M} \nabla_{\boldsymbol{R}}^{2}-\frac{\hbar^{2}}{2 \mu} \nabla_{\boldsymbol{r}}^{2}-g \mu_{\mathrm{B}} B\left(S_{1}^{z}+S_{2}^{z}\right)+V(r),
\end{aligned}
$$

where as before $M=m_{1}+m_{2}$ and $\mu=\frac{m_{1} m_{2}}{m_{1}+m_{2}}$. We insert the above wave function into the Hamiltonian and follow standard procedures, assuming that the potential $V_{\boldsymbol{k}, \boldsymbol{k}^{\prime}} \equiv \frac{N}{V} \int_{V} \mathrm{e}^{-\mathrm{i} \boldsymbol{k} \boldsymbol{r}} V(r) \mathrm{e}^{\mathrm{i} \boldsymbol{k}^{\prime} \boldsymbol{r}} \mathrm{d}^{3} r$ is attractive when all the particles composing Cooper pairs with momenta $(\boldsymbol{Q}, \boldsymbol{k})$ and $\left(\boldsymbol{Q}, \boldsymbol{k}^{\prime}\right)$ are in a region above the Fermi surface of width $\hbar \omega_{\mathrm{D}}$ (cf. Fig. 2). Having done that, we arrive at the new gap equation.

\subsection{Pair binding energy}

The gap equation at first sight looks very alike the standard one. The fundamental difference is the range of integration $\bar{W}_{\boldsymbol{Q}}$ (cf. Fig. 2 and the explanation below)

\footnotetext{
${ }^{\dagger}$ Throughout this paper we neglect the effect of the magnetic field on the orbital motion, as we consider weak-field limit only.
} 


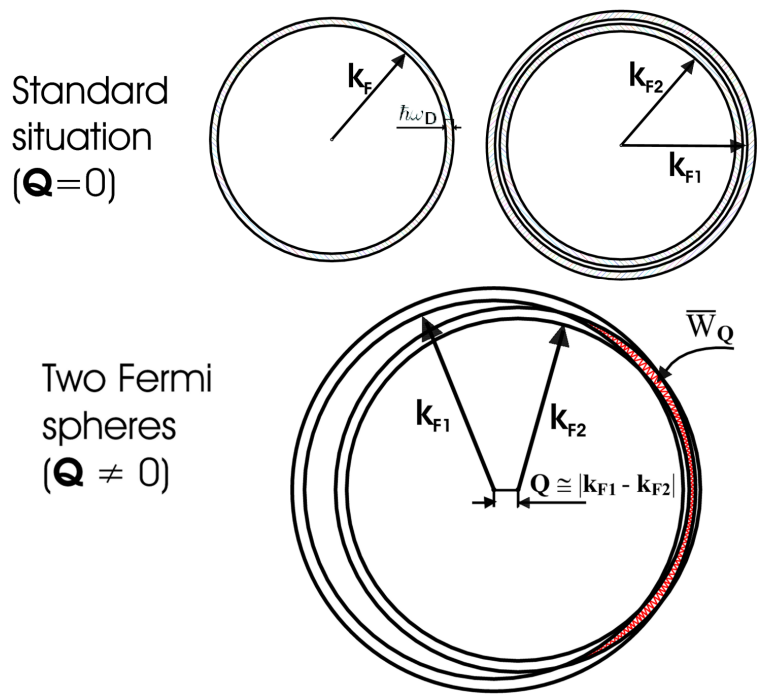

Fig. 2. The integration range $\bar{W}_{\boldsymbol{Q}}$, which is defined as the set $\bar{W}_{\boldsymbol{Q}} \equiv\left\{W_{1}\right\} \cap\left\{W_{2}+\right.$ $\boldsymbol{Q}\}-\frac{m_{1}}{M} \boldsymbol{Q}$, where $W_{i} \equiv\left\{\boldsymbol{k} \mid \frac{\hbar^{2} k^{2}}{2 m_{i}} \in\left[\epsilon_{\mathrm{F}}, \epsilon_{\mathrm{F}}+\hbar \omega_{\mathrm{D}}\right]\right\}$. By adding a vector to a region in reciprocal space we mean shifting this region. In effect, $\bar{W}_{\boldsymbol{Q}}$ is nonspherical, as marked.

$$
\frac{N}{V_{0}}=\sum_{\boldsymbol{k} \in \bar{W}_{\boldsymbol{Q}}} \frac{1}{\epsilon_{\boldsymbol{Q}}+\epsilon_{\boldsymbol{k}}-2 \epsilon_{\mathrm{F}}+\Delta} .
$$

Now, we make simplifications to the gap equation, so that it assumes a little more transparent form. Namely, by using the notation

$$
\left\{\begin{array}{l}
\delta \epsilon_{\boldsymbol{k}_{1}}=\epsilon_{\boldsymbol{k}_{1}}-\epsilon_{\mathrm{F}} \\
\delta \epsilon_{\boldsymbol{k}_{2}}=\epsilon_{\boldsymbol{k}_{2}}-\epsilon_{\mathrm{F}} \\
\epsilon_{\boldsymbol{Q}}+\epsilon_{\boldsymbol{k}}-2 \epsilon_{\mathrm{F}}=\epsilon_{\boldsymbol{k}_{1}}+\epsilon_{\boldsymbol{k}_{2}}-2 \epsilon_{\mathrm{F}}=\delta \epsilon_{\boldsymbol{k}_{1}}+\delta \epsilon_{\boldsymbol{k}_{2}}
\end{array}\right.
$$

we transform the gap equation to the form

$$
\frac{N}{V_{0}}=\sum_{\boldsymbol{k} \in \bar{W}} \frac{1}{\delta \epsilon_{\boldsymbol{k}_{1}}+\delta \epsilon_{\boldsymbol{k}_{2}}+\Delta} .
$$

What can be seen is that the regions in the reciprocal space, which contribute most to the pairing, are those for which $\delta \epsilon_{\boldsymbol{k}_{1}}+\delta \epsilon_{\boldsymbol{k}_{2}} \approx 0$, i.e., the particles are close to the corresponding Fermi surfaces. Therefore, we expect the pairing to be strong, when two conditions on the pairing interaction region $\bar{W}_{\boldsymbol{Q}}$ are fulfilled. First, for a large part of the interaction region, the particles are not far from their Fermi surfaces. Second, the region has a maximum possible volume. In our procedure we optimize the binding energy with respect to the pairing interaction region $\bar{W}_{\boldsymbol{Q}}$, which is fully characterized by the center-of-mass momentum $\boldsymbol{Q}$. Therefore, all we need to do is to search for the solution with the lowest energy by varying the magnitude of the center-of-mass momentum $\boldsymbol{Q}$. 


\section{Results}

In this section we present results of our numerical calculations. In Fig. 3 we plot the dependence of the pairing-potential magnitude $V_{0}$ on the center-of-mass momentum $\boldsymbol{Q}$ obtained directly using the gap Eq. (19) and selecting the value of the gap magnitude, as indicated. The dependence of binding energy $\Delta$ on the momentum $\boldsymbol{Q}$ for several values of $V_{0}$ is shown in Fig. 4 . These results are obtained by an interpolation method, because of the character of the gap equation (it is

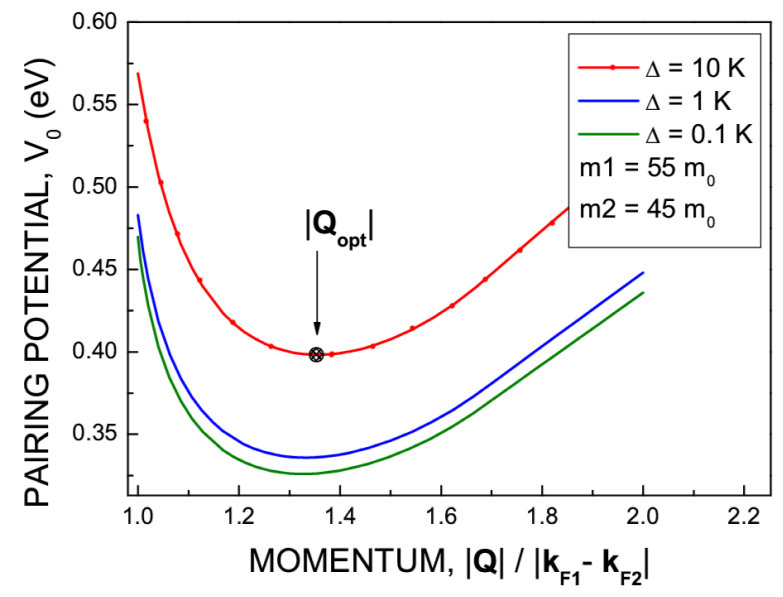

Fig. 3. Dependence of pairing potential magnitude $V_{0}$ on the center-of-mass momentum $\boldsymbol{Q}$ for selected values of the binding energy $\Delta$. $\boldsymbol{Q}_{\text {opt }}$ is the optimal value of momentum $Q$.

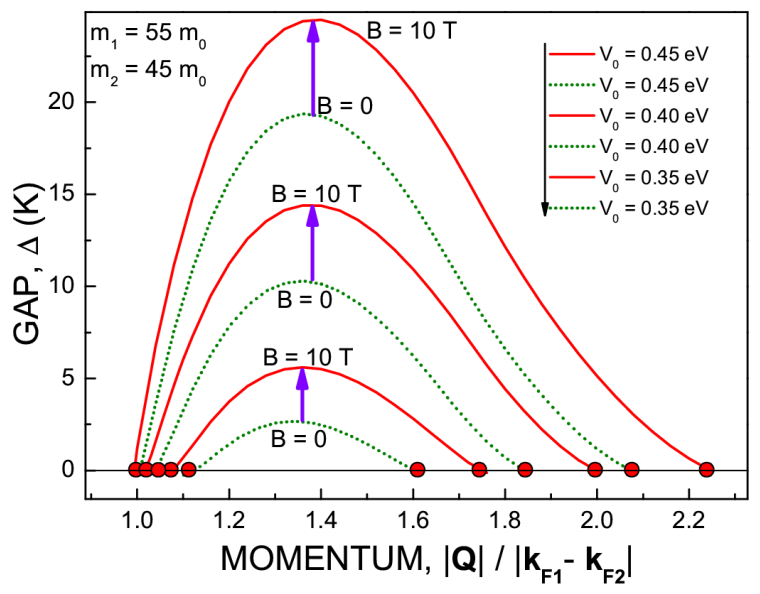

Fig. 4. Dependence of $\Delta$ on the pair momentum $Q$ for selected values of pairing-potential magnitude without applied field (dotted lines) and in the field of $10 \mathrm{~T}$ (solid lines). The masses are specified. 
easy to calculate $V_{0}$ for a given $\Delta$, but not the other way round). What can be seen from the figures is that the most energetically favorable solution is the one with nonzero center-of-mass momentum, approximately having the value

$$
Q \approx 1.4\left(k_{\mathrm{F} 1}-k_{\mathrm{F} 2}\right) \text {. }
$$

This is just because for such center-of-mass momentum $\boldsymbol{Q}$ the region in reciprocal space, where $\delta \epsilon_{\boldsymbol{k}_{1}}+\delta \epsilon_{\boldsymbol{k}_{2}} \approx 0$ is large, and the volume of the interaction region $\bar{W}_{\boldsymbol{Q}}$ do not reduce enough to destabilize the pairing (that actually happens for greater $\boldsymbol{Q}$, as marked by the dots).

It can be seen from Fig. 4 that the solution in the absence of magnetic field has a lower binding energy than the solution for $B=10 \mathrm{~T}$. The case of split masses without a magnetic field $\boldsymbol{B}$ is unrealistic. In real systems the presence of the field enforces the mass splitting, i.e. for stronger fields we would get a greater splitting and a smaller binding energy. The dependence of mass on magnetic field and its effect on pairing will be discussed elsewhere. Another characteristic feature is that for $V_{0} \lesssim 0.30 \mathrm{eV}$ the pair does not bind, which means that in the case of the split masses there exists a lower critical value of the potential needed to bind quasiparticles into a pair.

\section{Summary and outlook}

In this paper we have considered the Cooper problem with spin dependent masses of quasiparticles. Explicitly, we have analyzed the dependence of pair binding energy $\Delta$ on the pair momentum $\boldsymbol{Q}$, the state analogous to the FuldeFerrell-Larkin-Ovchinnikov phase (FFLO) in the applied magnetic field. The magnetic field was included only via Zeeman term. Next step is to include the spin dependent masses in a BCS-like theory, which would be quite similar to the FFLO formalism [6]. One could also consider ellipsoidal dispersion relations, which are relevant for quasi 2-dimensional systems with spin dependent masses, like $\mathrm{CeCoIn}_{5}$. A complete inclusion of the applied magnetic field (i.e. with the orbital effects incorporated) would also be an improvement, though we do not expect this task to be easy.

\section{Acknowledgments}

The work was supported by both Ministry of Higher Education and Science, grant No. 1 P03B 00129 and by the Foundation for Polish Science. The project was performed under the auspices of the COST P-16 network of European Science Foundation. The work presents a part of M.Sc. thesis of J.K., Jagiellonian University, Kraków 2007.

\section{References}

[1] L.N. Cooper, Phys. Rev. 104, 1189 (1956). See also: M. Randeria, J.-M. Duan, L.-Y. Shieh, Phys. Rev. B 41, 327 (1990); P. Wróbel, Z. Starypan, J. Kaczmarczyk, J. Spałek, Acta Phys. Pol. A 109, 541 (2006). 
[2] J. Spałek, P. Gopalan, Phys. Rev. Lett. 64, 2823 (1990).

[3] P. Korbel, J. Spałek, W. Wójcik, M. Acquarone, Phys. Rev. B 52, R2213 (1995); for a recent review see: J. Spałek, Phys. Status Solidi B 243, 78 (2006); Physica $B$ 378-380, 654 (2006).

[4] A. McCollam, S.R. Julian, P.M.C. Rourke, D. Aoki, T. Flouquet, Phys. Rev. Lett. 94, 186401 (2005).

[5] V.W. Liu, F. Wilczek, Phys. Rev. Lett. 90, 047002 (2003).

[6] P. Fulde, R.A. Ferrell, Phys. Rev. 135, A550 (1964); A.I. Larkin, Y.N. Ovchinnikov, Zh. Eksp. Teor. Fiz. 47, 1136 (1964) [Sov. Phys. JETP 20, 762 (1965)]. 